\section{OPEN ACCESS}

Edited by:

Ticiana A. Leal,

University of Wisconsin-Madison,

United States

Reviewed by:

Benjamin John Blyth,

Peter MacCallum Cancer

Centre, Australia

Oscar Arrieta,

National Institute of Cancerology

(INCAN), Mexico

*Correspondence:

Ellinor Peerschke

peersche@mskcc.org

Specialty section:

This article was submitted to

Thoracic Oncology,

a section of the journal

Frontiers in Oncology

Received: 27 April 2020

Accepted: 06 July 2020

Published: 12 August 2020

Citation:

Peerschke E, Stier K, Li X, Kandov E, de Stanchina E, Chang $Q$, Xiong $Y$, Manova-Todorova K, Fan N, Barlas A, Ghebrehiwet $B$ and Adusumilli PS (2020) gC1qR/HABP1/p32 Is a Potential New Therapeutic Target Against Mesothelioma. Front. Oncol. 10:1413. doi: 10.3389/fonc.2020.01413

\title{
gC1qR/HABP1/p32 Is a Potential New Therapeutic Target Against Mesothelioma
}

\section{Ellinor Peerschke ${ }^{1 *}$, Kenneth Stier ${ }^{2}$, Xiaoyu Li $^{3,4}$, Evelyn Kandov ${ }^{2}$, Elisa de Stanchina ${ }^{5}$, Qing Chang ${ }^{5}$, Yuquan Xiong ${ }^{4}$, Katia Manova-Todorova ${ }^{6}$, Ning Fan ${ }^{6}$, Afsar Barlas ${ }^{6}$, Berhane Ghebrehiwet ${ }^{2}$ and Prasad S. Adusumilli ${ }^{4}$}

${ }^{1}$ Department of Laboratory Medicine, Memorial Sloan Kettering Cancer Center, New York, NY, United States, ${ }^{2}$ Departments of Medicine and Pathology, Stony Brook University, Stony Brook, New York, NY, United States, ${ }^{3}$ Department of Thoracic Oncology, West China Hospital, Sichuan University, Chengdu, China, ${ }^{4}$ Department of Surgery, Thoracic Service, Memorial Sloan Kettering Cancer Center, New York, NY, United States, ${ }^{5}$ Memorial Sloan Kettering Cancer Center, Sloan Kettering Institute, New York, NY, United States, ${ }^{6}$ Molecular Cytology Core Facility, Sloan Kettering Institute, Memorial Sloan Kettering Cancer Center, New York, NY, United States

Mesothelioma is an aggressive cancer of the serous membranes with poor prognosis despite combination therapy consisting of surgery, radiotherapy, and platinum-based chemotherapy. Targeted therapies, including immunotherapies, have reported limited success, suggesting the need for additional therapeutic targets. This study investigates a potential new therapeutic target, gC1qR/HABP1/p32 (gC1qR), which is overexpressed in all morphologic subtypes of mesothelioma. $\mathrm{gC} 1 \mathrm{qR}$ is a complement receptor that is associated with several cellular functions, including cell proliferation and angiogenesis. In vitro and in vivo experiments were conducted to test the hypothesis that targeting gC1qR with a specific gC1qR monoclonal antibody 60.11 reduces mesothelioma tumor growth, using the biphasic mesothelioma cell line MSTO-211H (MSTO). In vitro studies demonstrate cell surface and extracellular gC1qR expression by MSTO cells, and a modest $25.3 \pm 1.8 \%(n=4)$ reduction in cell proliferation by the gC1aR blocking 60.11 antibody. This inhibition was specific for targeting the C1q binding domain of gC1qR at aa 76-93, as a separate monoclonal antibody 74.5.2, directed against amino acids 204-218, had no discernable effect. In vivo studies, using a murine orthotopic xenotransplant model, demonstrated an even greater reduction in MSTO tumor growth (50\% inhibition) in mice treated with the 60.11 antibody compared to controls. Immunohistochemical studies of resected tumors revealed increased cellular apoptosis by caspase 3 and TUNEL staining, in 60.11 treated tumors compared to controls, as well as impaired angiogenesis by decreased CD31 staining. Taken together, these data identify $\mathrm{gC} 1 \mathrm{qR}$ as a potential new therapeutic target against mesothelioma with both antiproliferative and antiangiogenic properties.

Keywords: mesothelioma, complement, gC1qR/HABP1/p32, monoclonal antibody therapy, therapeutic target 


\section{INTRODUCTION}

Mesothelioma is an aggressive cancer of the serous membranes, typically those lining the pleural space $(1,2)$. It is chiefly caused by exposure to and inhalation of asbestos. Treatment outcomes continue to be poor, despite multimodal therapy consisting of surgery, chemotherapy, and radiation (3-5). An estimated 38,400 individuals die globally each year from mesothelioma (6), and the incidence is expected to rise in the US as a result of asbestos exposure following destruction of the World Trade Center in New York, NY in 2001 (7). Novel therapies, especially targeted therapies, are needed to improve treatment outcomes and reduce off-target side effects $(8,9)$.

The complement system is emerging as a novel target in cancer therapy. Complement is involved not only in shaping the inflammatory tumor microenvironment, but also in tumor growth and spread (10). In this regard, the complement component $\mathrm{Clq}$ is increasingly recognized as a tumor-promoting factor. It has been reported to enhance cancer cell adhesion, migration, proliferation, and angiogenesis (11-13).

We have identified gC1qR (also known as p32/HABP1) as the major cellular binding site for C1q (14). Marked upregulation of $\mathrm{gClqR}$ expression has been observed in cancers of epithelial cell origin including breast, colon, and lung cancers $(15,16)$. In patients with breast cancer $(17,18)$, prostate cancer $(19)$, and serous ovarian adenocarcinoma (20), as well as endometrial cell cancer (21), overexpression of $\mathrm{gClqR}$ has been associated with poor prognosis. In addition, $\mathrm{gClqR}$ is being considered as a potential molecular target for delivery of cytotoxic agents $(22,23)$ in breast cancer.

$\mathrm{gClqR}$, is a multicompartmental cellular protein (24), with expression in mitochondria, the cytosol, and at the cell surface. In addition, $\mathrm{gClqR}$ is cleaved from cell membrane for release into the extracellular milieu by enzymes such as the membrane-type metalloproteinase $\operatorname{MT1MMP}(25,26)$. gC1qR shedding by cancer cells has been hypothesized to form a biochemical shield to protect malignant cells from complement mediated attack and produce inflammatory mediators to promote and enhance cancer metastasis (27). Interestingly, $\mathrm{gClqR}$ has been described to exert both pro-proliferative and antiproliferative properties in cancer (24). Many cancer cells have been reported to express $\mathrm{gClqR}$ with varying biological effects $(11,13,28)$.

We recently described the overexpression of $\mathrm{gClqR}$ in all mesothelioma subtypes, including epitheloid, sarcomatoid, and biphasic phenotypes (29). This finding suggests that gC1qR may represent a novel therapeutic target against mesothelioma. The present study tested this hypothesis in in vitro and in vivo experiments using a biphasic cultured human mesothelioma cell line, MSTO-211H (MSTO). The data demonstrate that targeting gC1qR with monoclonal antibody 60.11 reduces cell proliferation in vitro and tumor growth in vivo, associated with increased apoptosis and decreased angiogenesis, and provide proof of concept for further exploration of $\mathrm{gClqR}$ directed therapy in mesothelioma.

\section{METHODS}

\section{Materials}

The following materials were purchased from the sources indicated: MSTO-211H biphasic mesothelioma cell line (MSTO; ATCC, Manassas, VA); RPMI 1640, 100× penicillin/streptomycin, and $0.05 \%$ trypsin-EDTA (GIBCOInvitrogen, Grand Island, NY); heat-inactivated fetal bovine serum (FBS; Hyclone, Logan, UT); coating buffer (CB) comprised of $35 \mathrm{mM}$ sodium bicarbonate and $15 \mathrm{mM}$ sodium carbonate, aqueous; tris-buffered saline (TBS) comprised of $20 \mathrm{mM}$ tris- $\mathrm{HCl}, 150 \mathrm{mM}$ sodium chloride, and $0.05 \%$ tween, aqueous; ChromPure human IgG Fc fragments (Jackson ImmunoResearch, West Grove, PA); para-nitrophenyl phosphate (pNPP; Pierce, Rockford, IL); and Dulbecco's PBS (Mediatech Inc., Manassas, VA).

\section{Monoclonal Antibodies (mAbs)}

$\mathrm{mAbs}$ to gC1qR were generated as described (30). The 60.11 therapeutic antibody is directed against the $\mathrm{Clq}$ binding domain of gClqR, amino acids 76-93. $\mathrm{mAb}$ 74.5.2 recognizes amino acids 204-218, which constitutes the binding site for high molecular weight kininogen. AlexaFluor 488-conjugated secondary antibodies (Invitrogen, Carlsbad, CA) and nonimmune mouse IgG MOPC 21 (Sigma-Aldrich) were purchased.

\section{Cell Culture}

MSTO cells were cultured in RPMI supplemented with $10 \%$ fetal bovine serum, $100 \mathrm{U} / \mathrm{mL}$ penicillin, and $100 \mu \mathrm{g} / \mathrm{mL}$ streptomycin, in T175 culture flasks. Cultures were maintained at $37^{\circ} \mathrm{C}, 100 \%$ humidity, $5 \% \mathrm{CO}_{2}$, and subcultured when cells reached $\sim 90 \%$ confluence.

\section{Recombinant gC1qR}

The strategy for the construction of a plasmid containing the full-length (mature form or wild type, WT) and purification of the glutathione-S-transferase (GST)-gC1qR fusion products has been described in detail (14). The GST-gC1qR fusion product is cleaved by thrombin $(3.2 \mu \mathrm{g} / \mathrm{ml})$ and the GST-free $\mathrm{gClqR}$ protein is purified on fast protein liquid chromatography (FPLC, Pharmacia) using a Mono- $Q$ ion exchange column. The single peak containing the $\mathrm{gClqR}$ is pooled, concentrated to $1-2$ $\mathrm{mg} / \mathrm{ml}$, and stored at $-80^{\circ} \mathrm{C}$ in the presence of $50 \mathrm{nM}$ PPACK (D-phenylalanyl-L-prolyl-L-arginine chloromethyl ketone), a specific thrombin inhibitor (Sigma Aldrich).

\section{Detection of Soluble gC1qR}

A qualitative direct ELISA was used to evaluate the presence of soluble $\mathrm{gClqR}$ in MSTO culture medium. MSTO were seeded at 50,000 cells per well and grown for $48 \mathrm{~h}$. Culture supernatants were harvested and centrifuged to remove cellular debris, diluted 1:1 with $\mathrm{CB}$, and incubated in a high-binding microtiter well-plate for $1 \mathrm{~h}$ at ambient temperature. Wells were blocked with $1 \%$ heat-inactivated bovine serum albumin (BSA) in TBS, $10 \mathrm{~min}$. Immobilized $\mathrm{gClqR}$ was detected with biotinylated immunoaffinity purified polyclonal antibody to gClqR peptide (144-155) conjugated to alkaline phosphatase, and pNPP substrate. Absorbance was measured at $405 \mathrm{~nm}$. 
Soluble $\mathrm{gClqR}$ in pleural fluid from patients with advanced malignant pleural mesothelioma was quantified using a commercial, quantitative human gC1qR ELISA kit (Hycult, Netherlands) (26). Deidentified patient samples $(n=22)$ were evaluated according to manufacturer instruction and in compliance with Memorial Sloan Kettering IRB approved protocols (\#16-1547).

\section{Immunofluorescence Microscopy}

MSTO cells were seeded in 24-well-tissue culture-treated plates (50,000 cells per well) and grown to near-confluence. Cells were fixed $(10 \mathrm{~min})$ in $1 \%$ paraformaldehyde. Wells were blocked with $1 \% \mathrm{BSA}$ and $1 \mu \mathrm{g} / \mathrm{ml} \mathrm{Fc}$ fragments (30 min). gC1qR expression was examined by incubation (30 min) with anti-gC1qR mAb 60.11 (5 $\mu \mathrm{g}$ per well) followed by $30 \mathrm{~min}$ incubation with AlexaFluor 488-conjugated goat anti-mouse secondary antibody (5 $\mu$ g per well). Staining with non-immune rabbit IgG (NIRG) and AlexaFluor 488-conjugated goat anti-rabbit secondary antibody served as a negative control. DAPI $(0.2 \mu \mathrm{g}$ per well $)$ was used as a nuclear counterstain. Images were obtained using an Evos FL Imaging System at $10 \times$ magnification and normalized for background brightness. Additional brightness and contrast adjustments were applied uniformly to each image via Adobe Photoshop CS6.

\section{Flow Cytometry}

MSTO cells were detached from culture plates by incubation (30 min, room temperature) with $0.05 \%$ trypsin-0.01\% EDTA in $0.01 \mathrm{M}$ TBS, centrifuged $(800 \mathrm{~g}, 5 \mathrm{~min}$ ) and suspended using $10 \mathrm{mM}$ EDTA in PBS, pH 7.4. Cellular Fc receptors were blocked with $5 \mu \mathrm{g}$ Fc fragments per 500,000 cells. gC1qR expression was detected with mouse anti-gC1qR mAb 74.5.2, and MOPC 21, as negative control. The gC1qR 74.5.2 antibody was preferred for flow cytometry studies as it produced greater staining of cells than the 60.11 antibody. Primary antibodies were visualized with AlexaFluor 488-conjugated goat pAb to mouse IgG. Unstained cells were used an additional negative control. Cells were fixed in $1 \%$ paraformaldehyde. All reagents were diluted in DPBS. Fluorescence was determined using a FACSCalibur flow cytometer (BD Biosciences, San Jose, CA).

\section{MSTO Cell Adhesion Assay}

Cells were cultured as described above and imaged at 24-h intervals via compound light microscopy $(10 \times$ magnification $)$ with a PAXcam 3 microscope camera and Pax-it 11 software (Paxcam, Villa Park, IL). MSTO cells were seeded at 50,000 cells/well, in the presence or absence of $5 \mu \mathrm{g} / \mathrm{ml} \mathrm{recombinant}$ gC1qR. To evaluate the effect of immobilized gC1qR on cell adhesion, microtiter wells were incubated overnight $\left(37^{\circ} \mathrm{C}\right)$ with $20 \mu \mathrm{g} / \mathrm{ml}$ recombinant $\mathrm{gC} 1 \mathrm{qR}$ in $\mathrm{CB}$ and rinsed with $\mathrm{PBS}$ before exposure to cells.

\section{MSTO Cell Proliferation Assay}

MSTO cells were seeded in 24-well-cell culture plates at 50,000 cells per well and allowed to adhere overnight. Cultures were subsequently treated with $10 \mu \mathrm{g} / \mathrm{ml}$ anti-gC1qR mAb 60.11 or 74.5.2. Untreated cultures were used as controls. Cell proliferation was determined at $24 \mathrm{~h}$ intervals. At the desired times, cells were removed from culture wells using $0.05 \%$ trypsinEDTA, stained with trypan blue dye, and counted using a hemocytometer. Trypan blue positive cells were excluded from the count.

\section{Orthotopic Pleural Mesothelioma Mouse Model}

All procedures were performed under approved Institutional Animal Care and Use Committee protocols. Female severe combined immunodeficiency gamma mice (NSG (NOD, scid, gamma), Jackson Laboratories), 6-8 weeks old, were anesthetized using inhaled isoflurane and oxygen. Direct intrapleural injection of $1 \times 10^{6}$ GFP-Firefly Luciferase expressing MSTO-211H cells in $200 \mu \mathrm{l}$ serum-free media was administered to establish orthotopic malignant pleural mesothelioma tumors via a right thoracic incision, as previously described (31-33). For this proof of concept study, mice were divided into two treatment groups: vehicle $(n=10)$, and 60.11 antibody treatment $(n=10)(100$ $\mathrm{mg} / \mathrm{kg}$, administered twice weekly by intraperitoneal injection beginning on day 8 after tumor implantation). Intraperitoneal injection of antibody was chosen over the intravenous route, as it allowed for the administration of greater fluid volume, which was required to reach the desired therapeutic antibody dose. Clinical assessments of animal distress (weight loss, disruption of locomotor coordination, hunching, lack of grooming, lethargy) were made and recorded daily to assess toxicity.

Tumor burden was measured beginning on day 8 after tumor implantation, and at regular intervals until day 23 using quantitative bioluminescence imaging (BLI). Mice were imaged with the Xenogen IVIS 100 Imaging System, $20 \mathrm{~min}$ following injection of a single intraperitoneal dose of $150 \mathrm{mg} / \mathrm{kg}$ D-Luciferin. Images were acquired for 5-30s depending on signal strength. BLI data were analyzed using Living Image 2.60 software and BLI signal reported as total flux (Photons/s). Due to high tumor burden observed in the control group, the experiment was terminated at day 23. At time of sacrifice, blood was collected for cell counts and measurement of serum 60.11 antibody levels using a direct ELISA assay with immobilized recombinant gC1qR. Tumors were removed, fixed and processed for histologic (hematoxylin and eosin staining) and immunohistochemical evaluation.

\section{Immunohistochemical Analysis}

Tissue processing and immunohistochemical analysis was performed by the Molecular Cytology Core Facility of Memorial Sloan Kettering Cancer Center as previously described (31, 32). In brief, tissues were fixed in $4 \%$ formaldehyde and processed by paraffin embedding using a tissue processor (Leica ASP6025). Five micrometer sections were obtained and applied to superfrost plus slides. Immunohistochemical detection of Ki67, Cleaved Caspase 3, TUNEL, and CD31 was performed using a Discovery XT processor (Ventana Medical Systems). Slides were counterstained with hematoxylin and cover-slipped with Permount (Fisher Scientific). 


\section{Ki-67}

The Discovery XT autostainer was programmed to incubate slides with primary rabbit polyclonal Ki-67 antibody (Abcam, catalog \# ab16667) at $1 \mu \mathrm{g} / \mathrm{ml}$ for $4 \mathrm{~h}$, followed by incubation with secondary antibody (biotinylated goat anti-rabbit IgG; Vector labs) at a concentration of $5.75 \mu \mathrm{g} / \mathrm{ml}$ for 30 min. Blocker D, Streptavidin-HRP, and DAB detection kit (Ventana Medical Systems) were used according to manufacturer instructions.

\section{Cleaved Caspase 3}

A rabbit polyclonal Cleaved Caspase 3 antibody (Cell Signaling, catalog \# 9661) was used at $0.1 \mu \mathrm{g} / \mathrm{ml}$ concentration. Slides were incubated in the Discovery XT autostainer for $3 \mathrm{~h}$. Incubation with secondary antibody (biotinylated goat antirabbit IgG; Vector labs) at a concentration of $5.75 \mu \mathrm{g} / \mathrm{ml}$ occurred for 20 min. Blocker D, Streptavidin-HRP, and DAB detection kit (Ventana Medical Systems) were used according to manufacturer instructions.

\section{TUNEL}

Terminal deoxynucleotidyl dUTP nick end labeling (TUNEL) analysis was done as follows. Slides were manually deparaffinized in xylene, rehydrated in a series of alcohol dilutions (100, 95, and $70 \%)$ and tap water, and placed into the autostainer, where tissue sections were treated with Proteinase K $(20 \mu \mathrm{g} / \mathrm{ml}$ in PBS) for $8 \mathrm{~min}$, and incubated with endogenous biotin blocking kit (Roche) for $12 \mathrm{~min}$, followed by incubation with labeling mix: TdT (Roche, 1,000 U/ml) and biotin-dUTP (Roche, $4.5 \mathrm{nmol} / \mathrm{ml}$ ) for $2 \mathrm{~h}$. Detection was performed with Streptavidin-HRP and $\mathrm{DAB}$ detection kit (Ventana Medical Systems) according to the manufacturer's instruction.

\section{CD31}

Primary antibody, a rat anti-mouse CD31 antibody (Dianova, catalog \# DIA-310) was used at $2 \mu \mathrm{g} / \mathrm{ml}$. Slides were incubated in the autostainer for $6 \mathrm{~h}$, followed by exposure to biotinylated rabbit anti-rat IgG (Vector, 1:200 dilution) for $60 \mathrm{~min}$. Blocker D, Streptavidin-HRP, and DAB detection kit (Ventana Medical Systems) were used according to the manufacturer's instructions.

A
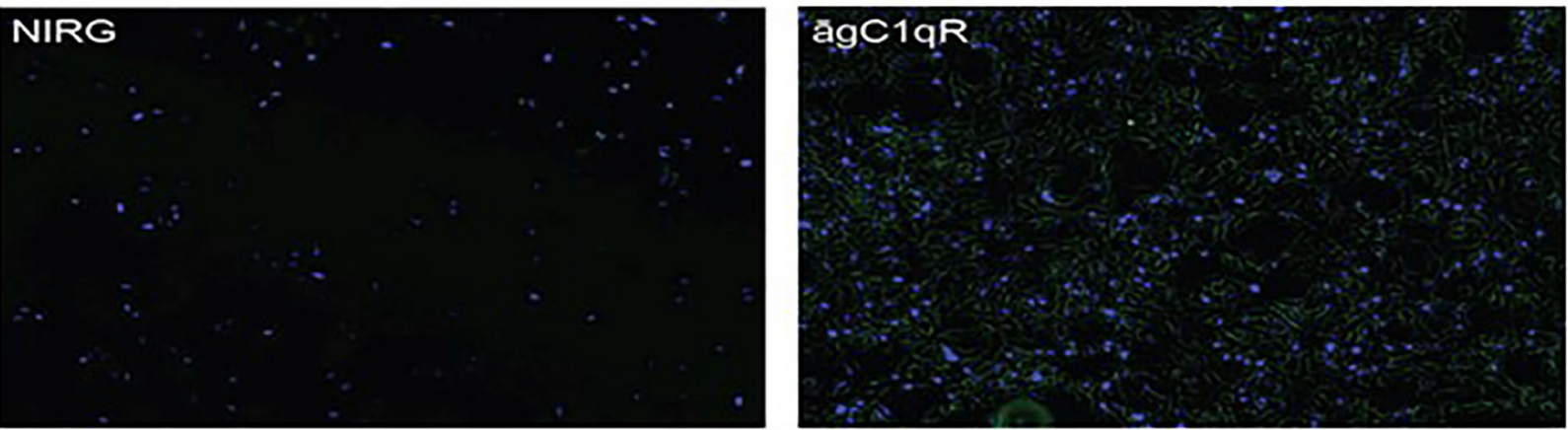

B

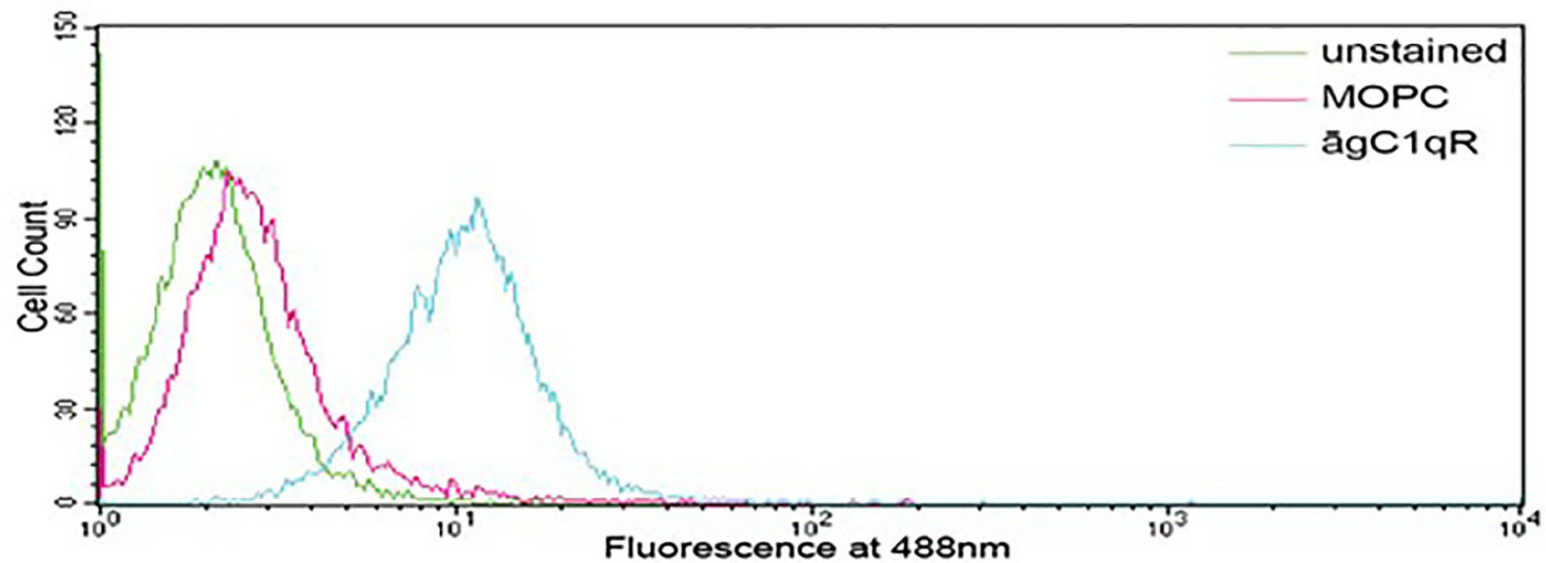

FIGURE 1 | Mesothelioma MSTO-211 H cells expresses gC1qR. (A) Immunofluorescence photomicrographs of stained MSTO-211H cells in culture. Results of a typical experiment. Non-permeabilized cell monolayers were stained with non-immune rabbit lgG (NIRG) or 60.11 anti gC1qR antibody (āgC1qR) and appropriate secondary antibodies conjugated to AlexaFluor 488 (green), as well as DAPI nuclear stain (blue). Cells were imaged with a fluorescence microscope at 10x magnification. The presence of $\mathrm{gC} 1 \mathrm{gR}$ is shown by green staining in the right image. (B) Flow cytometric analysis of MSTO-211H cell surface gC1qR expression. Non-permeabilized MSTO-211 H cells in suspension were stained with non-immune mouse lgG (MOPC) or anti gC1qR antibody (āgC1qR) and appropriate secondary antibodies conjugated to AlexaFluor 488. Stained and unstained cells were analyzed. Expression of cell surface gC1qR in a typical experiment is shown by an increase in fluorescence of cells treated with anti gC1qR antibody (blue curve). 


\section{Quantitative Analysis of Immunohistochemical Staining}

Quantitative analysis of immunohistochemical staining was performed of images generated by a slide scanner (Panoramic Flash 250, 3DHistech, Hungary) using Image J software.

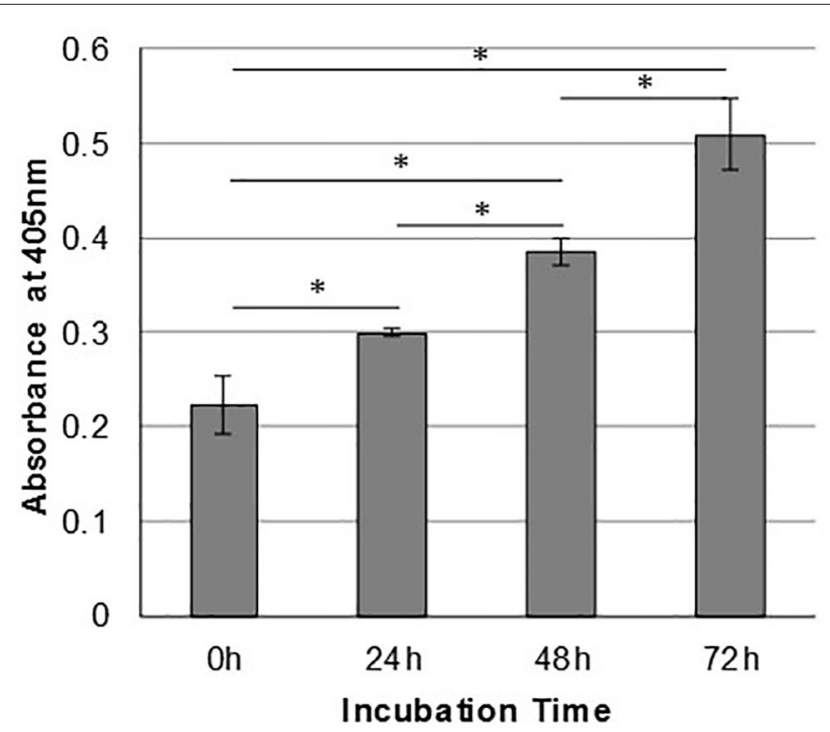

FIGURE 2 | Mesothelioma cells shed gC1qR into the extracellular milieu. MSTO-211H cells were cultured in complete medium, and supernatants were sampled at 24-h intervals. Relative concentrations of gC1qR in the supernatants at each time point were determined via a direct ELISA. Error bars represent mean substrate absorbance \pm standard deviation. $n=2$ separate experiments performed in duplicate. *Significantly different results at $p<0.05$.

\section{Statistical Analysis}

Data were analyzed with Student's $t$-test as applicable, and $p<0.05$ were considered statistically significant. All statistical analysis, calculations, and graphing were performed in Excel (Microsoft, Redmond, WA).

\section{RESULTS}

\section{In vitro Studies}

In order to assess the effect of anti-gC1qR therapy on MSTO cell proliferation, in vitro studies first evaluated the expression of the target antigen by MSTO cells. Expression of gC1qR by MSTO cells was demonstrated by immunofluorescence microscopy (Figure 1A) and flow cytometry (Figure 1B). In addition, soluble $\mathrm{gClqR}$ was detected in culture supernatants of MSTO cells. Progressively increasing amounts of $\mathrm{gClqR}$ were shed into the cell culture medium over a $72 \mathrm{~h}$ time course (Figure 2). Soluble $\mathrm{gClqR}$ was found also in vivo, in 10 of 22 pleural fluids from patients with malignant pleural mesothelioma, with a mean concentration of $1.11 \pm 0.57 \mathrm{ng} / \mathrm{ml}(n=10)$. Interestingly, soluble $\mathrm{gClqR}$ and immobilized $\mathrm{gClqR}$, enhanced MSTO cell adhesion in culture (Figure 3). Cell proliferation was reduced $25.3 \pm 1.8 \%(n=4)$ by targeting $\mathrm{gClqR}$ with $\mathrm{mAb}(60.11)$ (Figure 4). This inhibition was specific for $\mathrm{mAb} 60.11$, directed against amino terminal amino acids 76-93, representing the C1q binding domain (28). As illustrated in Figure 4, gClqR $\mathrm{mAb}$ 74.5.2, directed against aa 204-218, had a negligible effect $(2.9 \pm 2.2 \%$ inhibition).

\section{In vivo Studies}

An orthotopic mouse model of malignant pleural mesothelioma was used to evaluate $\mathrm{gClqR}$ blockade with $\mathrm{mAb} 60.11$ on MSTO cell proliferation. The data are summarized in Table 1. Animals

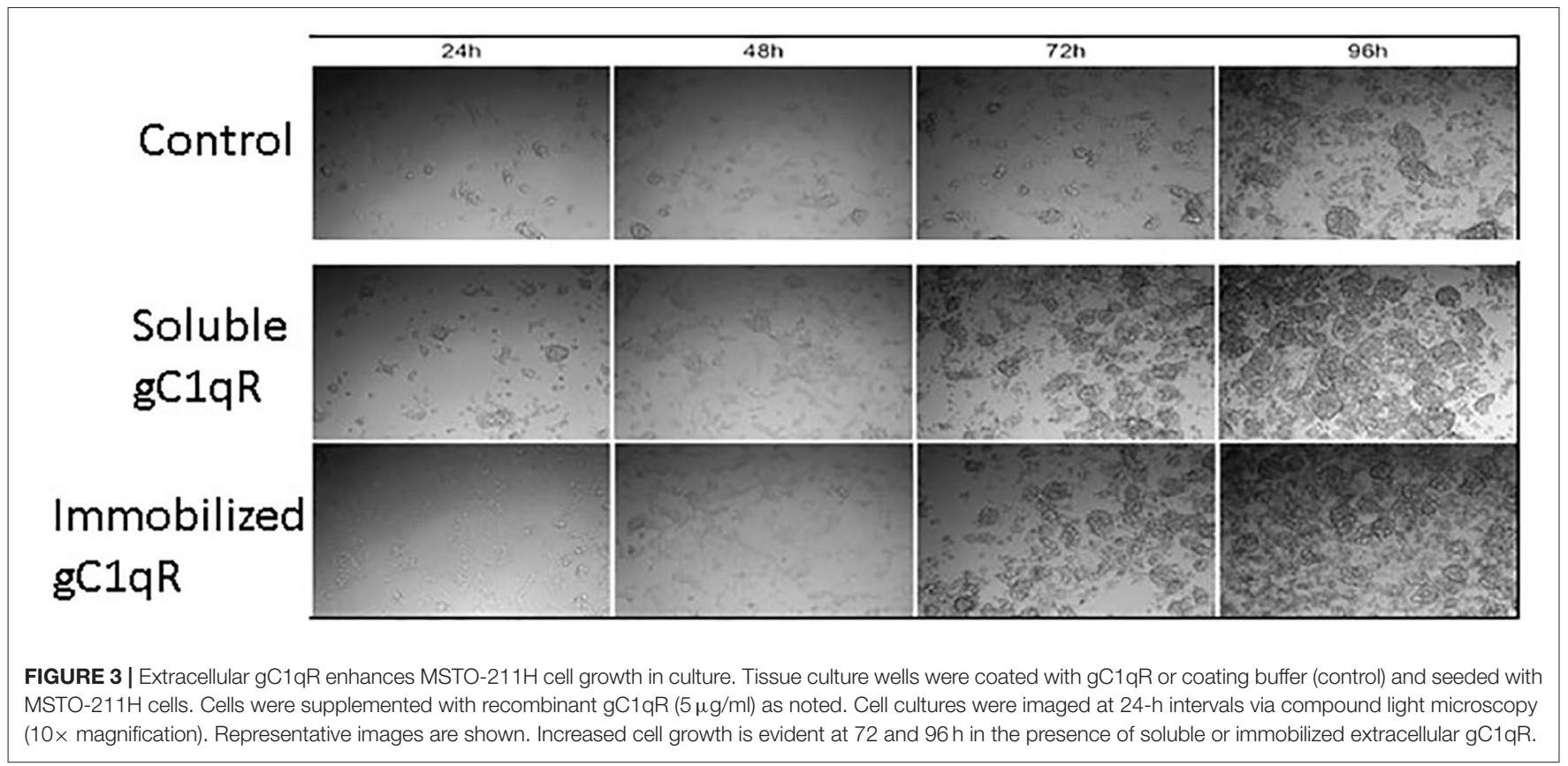




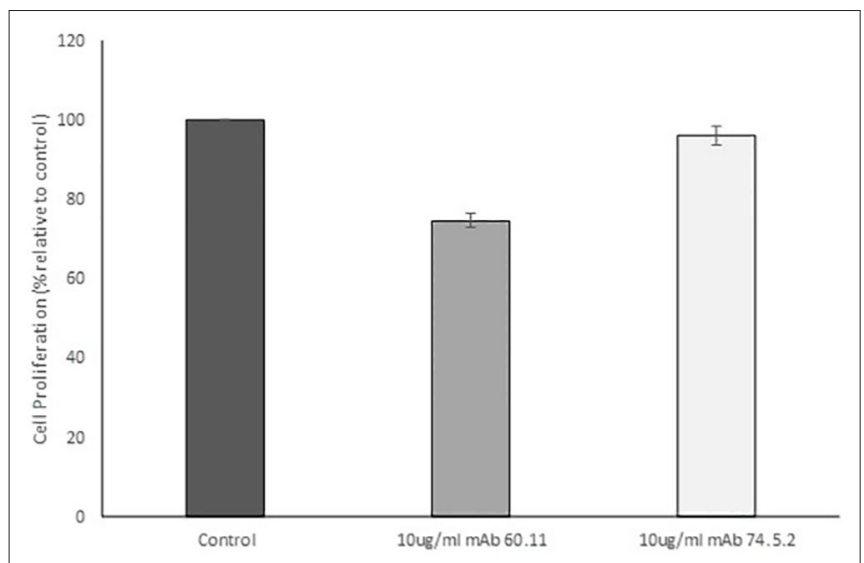

FIGURE 4 | Antibodies directed against the C1q binding site of gC1qR decrease mesothelioma cell proliferation. MSTO-211H cells was treated with $10 \mu \mathrm{g} / \mathrm{mL}$ monoclonal anti gC1qR antibody mAb 60.11 directed against the C1q binding region (aa 76-93) or mAb 74.5.2 directed against the high molecular weight kininogen binding domain (aa 204-218). Cell proliferation was determined via hemocytometer cell counts of viable cells after $96 \mathrm{~h}$ incubation. Error bars represent mean cell population \pm standard deviation. $n=2$ separate experiments performed in duplicate. *Significant difference from control at $p=1.33 \times 10^{-5}$.

TABLE 1 | Targeted gC1qR (60.11) treatment reduces MSTO-211H tumor cell growth in an orthotopic murine xenotransplant model.

\section{Treatment Group}

Treatment Group
Tumor Burden
$\left(\right.$ BLI, Total Flux, $\times$ 10 $\left.^{7}\right)$

\begin{tabular}{lccc} 
& Vehicle Control & $\mathbf{6 0 . 1 1}$ Treatment & $\boldsymbol{p}$ \\
\hline Baseline & $2.1 \pm 1.0$ & $2.4 \pm 1.4$ & 0.635 \\
Week 1 & $18.8 \pm 6.1$ & $9.6 \pm 3.6$ & 0.0006 \\
Week 2 & $61.1 \pm 25.6$ & $34.6 \pm 19.6$ & 0.018 \\
\hline
\end{tabular}

BLI, Bioluminescence Imaging.

treated with the 60.11 antibody showed an approximately $50 \%$ reduction in tumor development compared to vehicle control. Serum 60.11 antibody concentrations measured on day of sacrifice ranged from 30 to $50 \mu \mathrm{g} / \mathrm{ml}$. 60.11 therapy was not associated with clinical changes such as weight loss (Table 2), disruption of locomotor coordination, hunching, and lack of grooming or lethargy. Comparison of peripheral blood cell counts showed no change in RBC and platelet counts between treatment groups, but a modest decrease in WBC counts, predominantly associated with a decrease in neutrophils in 60.11 treated mice, was observed (Table 2).

Figure 5 shows representative images of tumors resected from control and 60.11 treated mice. Although tumors from 60.11 treated mice were macroscopically smaller than those excised from control mice, no histologic differences were appreciated by H\&E staining. Tumors consisted of densely packed MSTO cells with significant areas of necrosis. Immunohistochemical analysis (Figure 6) revealed an increase in early and late
TABLE 2 | Effect of 60.11 therapy on mouse weight and blood cell counts.

\begin{tabular}{lcc}
\hline & \multicolumn{2}{c}{ Treatment Groups } \\
\cline { 2 - 3 } & Vehicle & $\mathbf{6 0 . 1 1}$ treatment \\
\hline Weight $(g)$ & $23.0 \pm 1.16$ & $23.5 \pm 1.08$ \\
Cell Count & & \\
RBC $\left(10^{6} / \mu \mathrm{l}\right)$ & $9.01 \pm 0.70$ & $8.58 \pm 0.63$ \\
Hgb $(\mathrm{g} / \mathrm{dl})$ & $15.01 \pm 1.15$ & $14.23 \pm 1.01$ \\
HCT $(\%)$ & $43.54 \pm 3.14$ & $41.91 \pm 2.50$ \\
MCV $(\mathrm{fl})$ & $48.33 \pm 0.61$ & $48.92 \pm 1.13$ \\
Platelets $\left(10^{3} / \mu \mathrm{l}\right)$ & $1579 \pm 121$ & $1544 \pm 172$ \\
WBC $\left(10^{3} / \mu \mathrm{l}\right)$ & $5.02 \pm 1.05$ & $3.65 \pm 1.02^{*}$ \\
Neutrophils $\left(10^{3} / \mu \mathrm{l}\right)$ & $4.57 \pm 0.92$ & $3.24 \pm 0.91^{*}$ \\
Lymphocytes $\left(10^{3} / \mu \mathrm{l}\right)$ & $0.032 \pm 0.019$ & $0.014 \pm 0.014^{*}$ \\
Monocytes $\left(10^{3} / \mu \mathrm{l}\right)$ & $0.292 \pm 0.134$ & $0.258 \pm 0.10$ \\
Eosinophils $\left(10^{3} / \mu \mathrm{l}\right)$ & $0.125 \pm 0.067$ & $0.137 \pm 0.081$ \\
Basophils $\left(10^{3} / \mu \mathrm{l}\right)$ & $0.06 \pm 0.013$ & $0.02 \pm 0.004$ \\
\hline
\end{tabular}

${ }^{*} P<0.05$.

apoptosis markers, cleaved caspase 3 , and TUNEL, respectively, in tumors from 60.11 treated mice compared to controls. No difference in the tumor cell proliferation index (Ki67) was noted between treatment groups. Interestingly, 60.11 therapy was associated with decreased tumor CD31 staining, suggesting decreased angiogenesis. In addition, the CD31 positive vessels in tumors of 60.11 treated animals appeared generally small and poorly developed.

\section{DISCUSSION}

The data provide proof of concept that targeting the complement receptor, $\mathrm{gClqR}$, at the $\mathrm{Clq}$ binding site, may provide a potential novel therapeutic strategy in mesothelioma. The present study was developed based on our recent observation that $\mathrm{gClqR}$ is overexpressed in malignant pleural mesothelioma (29), and reports from breast and lung cancer models, indicating decreased tumor cell proliferation (34-36) using gC1qR-targeted strategies. In vitro studies with the biphasic mesothelioma cell line MSTO demonstrate the presence of targetable $\mathrm{gClqR}$ on the cell surface and in the extracellular milieu. Interestingly, extracellular gC1qR enhanced MSTO cell adhesion and proliferation in vitro, and may have similar direct effects in vivo. Additional postulated roles for extracellular gC1qR include shielding of the tumor from classical complement mediated attack, and activation of the kallikrein system with bradykinin generation and vascular leakage that may contribute to tumor metastasis (27).

The ability of tumor cells to adhere to tissue surfaces is a key element in metastasis formation (37). Thus, the enhanced ability of MSTO cells to adhere to immobilized $\mathrm{gC1qR}$ supports the hypothesis that $\mathrm{gClqR}$ in the tumor microenvironment may contribute to tumor progression via autocrine or paracrine effects. This observation requires further 


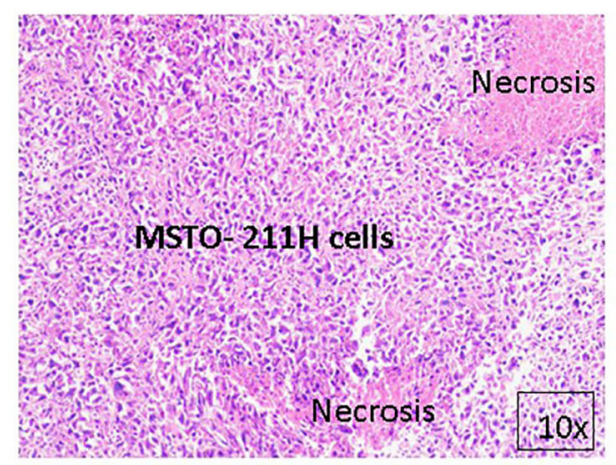

Vehicle Control

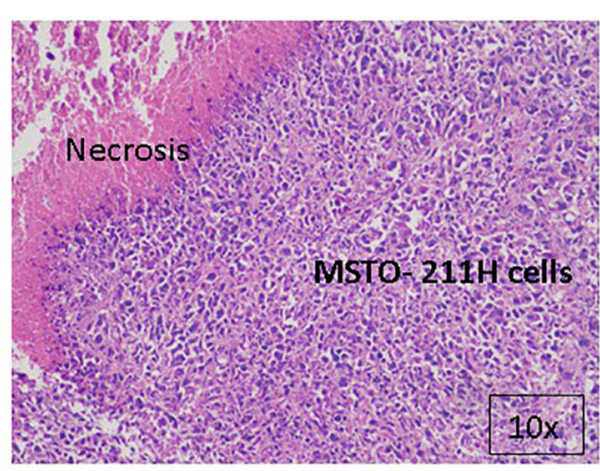

60.11 treated

FIGURE 5 | MSTO-211H tumors resected from control and 60.11 treated mice are histologically similar. Representative histologic (10x) images of tumors stained with hematoxylin and eosin show tightly packed MSTO-211 $\mathrm{H}$ cells and areas of necrosis. No histologic differences in tumor morphology were apparent between control and treatment groups.

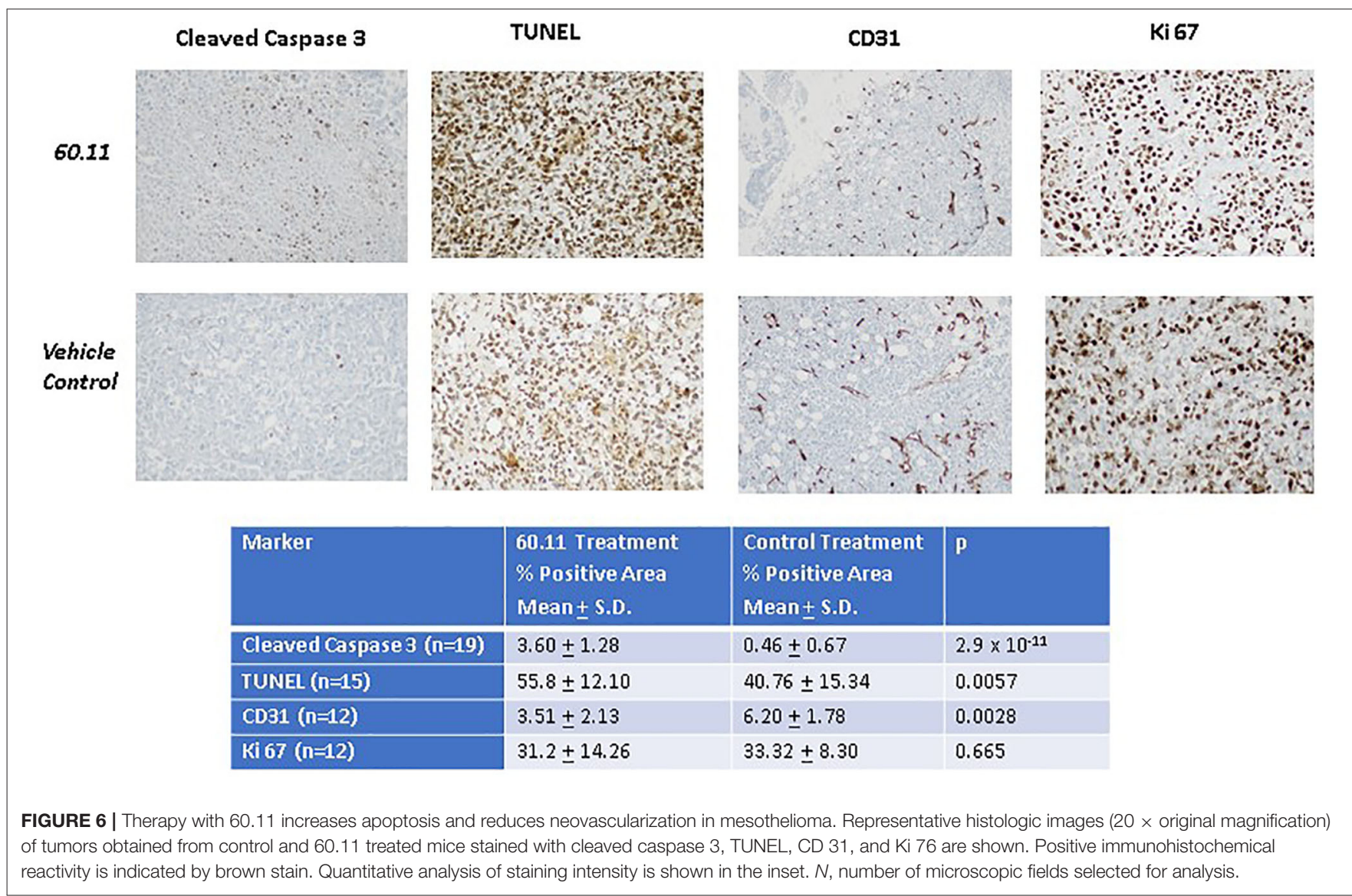

study, since levels of soluble $\mathrm{gClqR}$, previously detected in blood and body fluids (26), as well as those noted in pleural effusions from patients with MPM, are significantly lower than those found to enhance cell proliferation in vitro. Concentrations in the tumor microenvironment, however, are likely different, and greater in the microenvironment immediately adjacent to the tumor.

Targeting $\mathrm{gClqR}$, at the $\mathrm{Clq}$ binding site with $\mathrm{mAb}$ 60.11, reduced MSTO cell proliferation in vitro, and to a greater extent in vivo, using a clinically relevant orthotopic 
pleural mesothelioma mouse model. This model resembles pleural mesothelioma in humans with associated extensive lymphangiogenesis, regional invasion, and shortened survival (31). Data from immunohistochemical studies comparing tumors from 60.11 treated and control mice reveal that inhibition of in vivo mesothelioma tumor growth is associated with both increased apoptosis and decreased angiogenesis.

One of the major ligands of $\mathrm{gClqR}$ is $\mathrm{Clq}$ (14). The role of $\mathrm{Clq}$ in tumorigenesis is multifactorial. $\mathrm{Clq}$ is thought to promote tumor growth in part through its support of neovascularization (12). Indeed, in the present study, immunohistochemical analysis of tumors treated with 60.11 therapy demonstrate a decreased and abnormal microvasculature. Since mAb 60.11 is directed specifically against the $\mathrm{C} 1 \mathrm{q}$ binding site of $\mathrm{gC1qR}$, these findings support the hypothesis that $\mathrm{gClqR}-\mathrm{Clq}$ interactions in the tumor cell microenvironment contribute to mesothelioma tumor growth. Interestingly, recent immunohistochemical studies report the presence of $\mathrm{C1q}$ in mesothelioma (38). The observed anti-angiogenic effect of 60.11 therapy likely contributes to the overall in vivo effect of 60.11 therapy. This observation illustrates the complexity of tumor growth in vivo, and demonstrates that cell proliferation in vitro is not directly comparable to tumor growth in vivo.

Therapy with 60.11 was not associated with clinically discernable toxicity. Animal weights were similar between treatment and control groups. No difference in animal appearance, habitus or behavior was noted. However, a statistically significant decrease in total WBC was observed in the treatment group, which was attributed predominantly to a decrease in neutrophil count. Lymphocyte counts in the NSG mice are extremely low and differences between groups are therefore difficult to interpret. Given the observed significant anti-mesothelioma effect of 60.11 therapy, further exploration of both its therapeutic potential and toxicity profile are indicated.

The paucity of effective therapies for mesothelioma, including the limited success of mesothelin targeted therapy, immune checkpoint blockade, anti-angiogenesis therapies, and neoantigen based vaccines (39), necessitate the development of additional therapeutic strategies. This exploratory study provides the first in vivo proof of concept that targeting gC1qR at the C1q binding site can significantly reduce mesothelioma tumor burden by increasing tumor cell apoptosis and decreasing tumor angiogenesis. The study is limited by use of a single cell line and a single anti gC1qR targeting strategy. However, current results support further exploration of $\mathrm{gClqR}$ as a potential new therapeutic target. Investigation of additional $\mathrm{gC} 1 \mathrm{qR}$ targeting antibodies may further maximize treatment efficacy. For example, the 74.5.2 antibody, which has no effect on cell proliferation, is a potent inhibitor of vascular permeability,

\section{REFERENCES}

1. Mossman BT, Churg A. Mechanisms in the pathogenesis of asbestosis and silicosis. Am J Respir Crit Care Med. (1998) 157:1666-80. doi: $10.1164 /$ ajrccm.157.5.9 blocking the binding of high molecular weight kininogen to vascular endothelial cells (40). In addition, combining gC1qR targeted therapy with chemotherapy or other targeted therapies should be evaluated in further preclinical studies.

\section{DATA AVAILABILITY STATEMENT}

The raw data supporting the conclusions of this article will be made available by the authors, without undue reservation.

\section{ETHICS STATEMENT}

The studies involving human participants were reviewed and approved by Institutional Review Board/Privacy Board (FWA00004998). Written informed consent for participation was not required for this study in accordance with the national legislation and the institutional requirements. The animal study was reviewed and approved by Institutional Animal Care and Use Committee of Memorial Sloan Kettering Cancer Center (protocol no. 04-03-009).

\section{AUTHOR CONTRIBUTIONS}

EP, BG, and PA designed the study, supervised experiments, analyzed data, and wrote the manuscript. ES, QC, and YX conducted the in vivo studies. KM-T, NF, and $\mathrm{AB}$ performed immunohistochemical analyses. KS and EK performed in vitro studies. All authors participated in data review and review of the manuscript.

\section{FUNDING}

This work was supported by the Mesothelioma Applied Research Foundation (Award No. 549976), grants from the National Institutes of Health (P30 CA008748, R01 CA236615-01, and R01 CA235667), the National Institute of Allergy and Infectious Diseases (RO1 AI 060866 and RO1 AI-084178), the U.S. Department of Defense (BC132124, LC160212, CA170630, and CA180889), the Batishwa Fellowship, the Comedy vs. Cancer Award, the Druckenmiller Center for Lung Cancer Research Fellowship, the Emerson Collective Cancer Research Fund, the Esophageal Cancer Education Fund, the Memorial Sloan Kettering Technology Development Fund, the Miner Fund for Mesothelioma Research, the Mr. William H. Goodwin and Alice Goodwin, the Commonwealth Foundation for Cancer Research, and the Experimental Therapeutics Center of Memorial Sloan Kettering Cancer Center, and the Simons Summer Research Program funded by the Simons Foundation.
2. Adusumilli PS. Translational immunotherapeutics: chemoimmunotherapy for malignant pleural mesothelioma. Cancer. (2014) 120:326871. doi: $10.1002 / \mathrm{cncr} .28883$

3. Krug LM, Pass HI, Rusch VW, Kindler HL, Sugarbaker DJ, Rosenzweig $\mathrm{KE}$, et al. Multicenter phase II trial of neoadjuvant pemetrexed plus cisplatin followed by extrapleural pneumonectomy 
and radiation for malignant pleural mesothelioma. J Clin Oncol. (2009) 27:3007-13. doi: 10.1200/JCO.2008.20.3943

4. Nelson DB, Rice DC, Niu J, Atay S, Vaporciyan AA, Antonoff $\mathrm{M}$, et al. Long-term survival outcomes of cancer-directed surgery for malignant pleural mesothelioma: Propensity score matching analysis. J Clin Oncol. (2017) 35:3354-62. doi: 10.1200/JCO.2017. 73.8401

5. Vogelzang NJ, Rusthoven JJ, Symanowski J, Denham C, Kaukel E, Ruffie P, et al. Phase III study of pemetrexed in combination with cisplatin versus cisplatin alone in patients with malignant pleural mesothelioma. J Clin Oncol. (2003) 21:2636-44. doi: 10.1200/JCO.200 3.11 .136

6. Odgerel C-O, Takahashi K, Sorahan T, Driscoll T, Fitzmaurice C, Yoko$\mathrm{O} \mathrm{M}$, et al. Estimation of the global burden of mesothelioma deaths from incomplete national mortality data. Occup Environ Med. (2017) 74:8518. doi: 10.1136/oemed-2017-104298

7. Lioy PJ, Weisel CP, Millette JR, Eisenreich S, Vallero D, Offenberg J, et al. Characterization of the dust/smoke aerosol that settled east of the World Trade Center (WTC) in lower Manhattan after the collapse of the WTC 11 September 2001. Environ Health Perspect. (2002) 110:70314. doi: $10.1289 /$ ehp. 02110703

8. Scott AM, Allison JP, Wolchok JD. Monoclonal antibodies in cancer therapy. Cancer Immun. (2012) 12:14. doi: 10.1038/nrc3236

9. Drewe E, Powell RJ. Clinically useful monoclonal antibodies in treatment. J Clin Pathol. (2002) 55:81-5. doi: 10.1136/jcp.55.2.81

10. Kourtzelis I, Rafail S. The dual role of complement in cancer and its implication in anti-tumor therapy. Ann Transl Med. (2016) 4:265. doi: $10.21037 / \mathrm{atm} .2016 .06 .26$

11. Bulla R, Tripodo C, Rami D, Ling GS, Agostinis C, Guarnotta C, et al. $\mathrm{Cl} \mathrm{q}$ acts in the tumor microenvironment as a cancer-promoting factor independently of complement activation. Nat Commun. (2016) 7:10346 doi: $10.1038 /$ ncomms 10346

12. Bossi F, Tripodo C, Rizzi L, Bulla R, Agostinis C, Guarnotta C, et al. C1q as a unique player in angiogenesis with therapeutic implication in wound healing. Proc Natl Acad Sci U S A. (2014) 111:4209-14. doi: 10.1073/pnas.1311 968111

13. Ghebrehiwet B, Hosszu KK, Valentino A, Peerschke EIB. The C1q family of proteins: insights into the emerging non-traditional functions. Front Immunol. (2012) 3:52. doi: 10.3389/fimmu.2012. 00052

14. Ghebrehiwet B, Lim BL, Peerschke EIB, Willis AC, Reid KBM. Isolation, cDNA cloning, and overexpression of a $33-\mathrm{kDA}$ cell surface glycoprotein that binds to the globular heads of Clq. J Exp Med. (1994) 179:180921. doi: $10.1084 /$ jem.179.6.1809

15. Rubinstein DB, Stortchevoi A, Boosalis M, Ashfaq R, Ghebrehiwet B, Peerschke EI, et al. Receptor for the globular heads of $\mathrm{Clq}$ (gC1q$\mathrm{R}$, p33, hyaluronan binding protein) is preferentially expressed by adenocarcinoma cells. Intern J Cancer. (2004) 110:741-50. doi: 10.1002/ijc. 20105

16. Dembitzer FR, Kinoshita Y, Burstein D, Phelps RG, Beasley MB, Garcia $\mathrm{R}$, et al. $\mathrm{gClqR}$ expression in normal and pathologic human tissues: differential expression in tissues of epithelial and mesenchymal origin. J Histochem Cytochem. (2012) 60:467-74. doi: 10.1369/0022155412 440882

17. Chen YB, Jiang CT, Zhang GQ, Wang JS, Pang D. Increased expression of hyaluronic acid binding protein 1 is correlated with poor prognosis in patients with breast cancer. J Surg Oncol. (2009) 100:382-6. doi: 10.1002/jso. 21329

18. Jiang $\mathrm{Y}, \mathrm{Wu} \mathrm{H}$, Liu J, Chen $\mathrm{Y}$, Xie J, Zhao Y, et al. Increased breast cancer risk with $\mathrm{HABP} 1 / \mathrm{p} 32 / \mathrm{gC} 1 \mathrm{qR}$ genetic polymorphism rs2285747 and its upregulation in northern Chinese women. Oncotarget. (2017) 8:1393241. doi: 10.18632/oncotarget. 14737

19. Amamoto $R$, Yagi $M$, Song $Y$, Oda $Y$, Tsuneyoshi $M$, Naito $S$, et al. Mitochondrial p32/C1QBP is highly expressed in prostate cancer and is associated with shorter prostate-specific antigen relapse time after radical prostatectomy. Cancer Sci. (2011) 102:639-47. doi: 10.1111/j.1349-7006.2010.01828.x
20. Yu G, Wang J. Significance of hyaluronan binding protein (HABP1/P32/gC1qR) expression in advanced serous ovarian cancer patients. Exp Mol Pathol. (2013) 94:210-5. doi: 10.1016/j.yexmp.2012.06.007

21. Zhao J, Liu T, Yu G, Wang J. Overexpression of HABP1 correlated with clinicopathological characteristics and unfavorable prognosis in endometrial cancer. Tumour Biol. (2015) 36:1299-306. doi: 10.1007/s13277-014-2761-8

22. Fogal V, Zhang L, Krajewski S, Ruoslathi E. Mitochondria/cell surface protein $\mathrm{p} 32 / \mathrm{gClqR}$ as a molecular target in tumor cells and tumor stroma. Cancer Res. (2008) 7210-8. doi: 10.1158/0008-5472.CAN-0 7-6752

23. Paasonen L, Sharma S, Braun GB, Katamraju VR, Chung TDY, She Z, et al. New p32/gC1qR ligands for targeted drug delivery. Chembiochem. (2016) 17:570-5. doi: $10.1002 /$ cbic. 201500564

24. Saha P, Datta K. Multifunction, multicompartmental hyaluronan-binding protein 1 (HABP1/p32/gC1qR: Implication in cancer progression and metastasis. Oncotarget. (2018) 9:10784-807. doi: 10.18632/oncotarget. 24082

25. Rozanov DV, Ghebrehiwet B, Postnova TI, Eichinger A, Deryugina EI, Strongin AY. The hemopexin-like C-terminal domain of membrane type 1 matrix metalloproteinase regulates proteolysis of a multifunctional protein, gC1qR. J Biol Chem. (2002) 277:9318-25. doi: 10.1074/jbc.M110 711200

26. Peerschke EIB, Brandwijk RJMGE, Dembitzer FR, Kinoshito Y, Ghebrehiwet B. Soluble gC1qR in blood and body fluids: examination in a pancreatic cancer patient cohot. Int J Cancer Res Mol Mech. (2015) 1:10. doi: $10.16966 / 2381-3318.110$

27. Peerschke EIB, Ghebrehiwet B. $\mathrm{cClqR} / \mathrm{CR}$ and $\mathrm{gC} 1 \mathrm{qR} / \mathrm{p} 33$ : Observations in cancer. Mol Immunol. (2014) 61:100-9. doi: 10.1016/j.molimm.2014 .06 .011

28. Ghebrehiwet B, Jesty J, Xu S, Vinayagasundaram R, Vinayagasundaram U, Ji Y, et al. Structure-function studies using deletion mutants identify domains of $\mathrm{gClqR} / \mathrm{p} 33$ as potential therapeutic targets for vascular permeability and inflammation. Front Immunol. (2011) 2:58. doi: 10.3389/fimmu.201 1.00058

29. Li X, Eguchi T, Aly RG, Chintala NK, Tan KS, Zauderer, et al. Globular $\mathrm{C} 1 \mathrm{q}$ receptors $(\mathrm{gClqR} / \mathrm{p} 32 / \mathrm{HABP} 1)$ is overexpressed in malignant pleural mesothelioma and is associated with increased survival in surgical patients treated with chemotherapy. Front Oncol. (2019) 9:1042. doi: 10.3389/fonc.2019.01042

30. Ghebrehiwet B, Lu PD, Zhang W, Lim BL, Eggleton P, Leigh $\mathrm{LE}$, et al. Identification of functional domains on $\mathrm{gC1Q}-\mathrm{R}$, a cell surface protein that binds to the globular "heads" of $\mathrm{C} 1 \mathrm{Q}$, using monoclonal antibodies and synthetic peptides. Hybridoma. (1996) 15:333-42. doi: 10.1089/hyb.1996.15.333

31. Servais EL, Suzuki K, Colovos C, Rodriguez L, Sima C, Fleisher M, et al. An in vivo platform for tumor biomarkder assessment. PLoS One. (2011) 6:e26722. doi: 10.1371/journal.pone.0026722

32. Servais EL, Colovos C, Rodriguez L, Bograd AJ, Nitadori J, Sima C, et al. Mesothelin overexpression promotes mesothelioma cell invasion and MMP-9 secretion in an orthotopic mouse model and in epithelioid pleural mesothelioma patients. Clin Cancer Res. (2012) 18:2478-89. doi: 10.1158/1078-0432.CCR-11-2614

33. Adusumilli PS, Cherkassky L, Villena-Vargas J, Colovos C, Servais E, Plotkin J, et al. Regional delivery of mesothelin-targeted CAR T cell therapy generates potent and long-lasting $\mathrm{CD} 4$-dependent tumor immunity. Sci Transl Med. (2014) 6:261ra51. doi: 10.1126/scitranslmed.3 010162

34. Kandov E, Kaur A, Kishore U, Ji P, Williams J, Peerschke EIB, et al. $\mathrm{C} 1 \mathrm{q}$ and $\mathrm{C} 1 \mathrm{q}$ receptors $(\mathrm{gClqR}$ and $\mathrm{cClqR}$ ) as potential novel targets for therapy against breast cancer. Curr Trends Immunol. (2018) 19:59-76. Available online at: https://www.scopus.com/inward/ record.uri?eid $=2$ - $s 2.0-85062166884$ \&partnerID $=40 \& \mathrm{md} 5=$ 7 cbdf37967cb340d8d1f6a75773c4557

35. Kim KB, Yi JS, Nguyen N, Lee JH, Kwon YC, Ahn BY, et al. Cell-surface receptor for complement component $\mathrm{C} 1 \mathrm{q}(\mathrm{gClqR})$ is a key regulator for lamellipodia formation and cancer metastasis. J Biol Chem. (2011) 286:23093101. doi: $10.1074 /$ jbc.M111.233304 
36. Kim B-C, Hwang H-J, An H-T, Lee H, Park JS, Hong J, et al. Antibody neutralization of cell-surface gC1qR/HABP1/SF2-p32 prevents lamellipodia formation and tumorigenesis. Oncotarget. (2016) 7:4997285. doi: 10.18632/oncotarget.10267

37. Varani J, Lovett EJ, Elgebaly S, Lundy J, Ward PA. In vitro and in vivo adherence of tumor cell variants correlated with tumor formation. Am J Pathol. (1980) 101:345-52.

38. Agostinis C, Vidergar R, Belmonte B, Mangogna A, Amadio L, Geri P, et al. Complement protein $\mathrm{C} 1 \mathrm{q}$ binds to hyaluronic acid in the malignant pleural mesothelioma microenvironment and promotes tumor growth. Front Immunol. (2017) 8:1559. doi: 10.3389/fimmu.2017.01559

39. Mutti L, Peikert T, Robinson BWS, Scherpereel A, Tsao AS, de Perrot M, et al. Scientific advances and new frontiers in mesothelioma therapeutics. J Thorac Oncol. (2018) 13:1269-83. doi: 10.1016/j.jtho.2018.06.011

40. Fandaros M, Ong CL, Rubenstein DA, Joseph K, Kaplan AP, Yin W, et al. Angioedema and shear stress modulate endothelial permeability through gC1qR. FASEB J. (2019) 33(Suppl 1):542.15.
Conflict of Interest: $\mathrm{EP}$ and $\mathrm{BG}$ receive royalty income from the commercialization of $\mathrm{gClqR}$ antibodies, including 60.11, and hold a licensed patent for the use of $\mathrm{gClqR}$ antibodies. PA has received research funding from ATARA Biotherapeutics and OSE Immunotherapies.

The remaining authors declare that the research was conducted in the absence of any commercial or financial relationships that could be construed as a potential conflict of interest.

Copyright (c) 2020 Peerschke, Stier, Li, Kandov, de Stanchina, Chang, Xiong, ManovaTodorova, Fan, Barlas, Ghebrehiwet and Adusumilli. This is an open-access article distributed under the terms of the Creative Commons Attribution License (CC BY). The use, distribution or reproduction in other forums is permitted, provided the original author(s) and the copyright owner(s) are credited and that the original publication in this journal is cited, in accordance with accepted academic practice. No use, distribution or reproduction is permitted which does not comply with these terms. 